\title{
Fast and Sensitive Radiochemical Method for Sr-90 Determination in Food and Feed by Chromatographic Extraction and Liquid Scintillation Counting
}

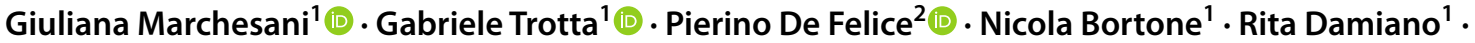 \\ Michele Nicolini ${ }^{1} \cdot$ Rosario Accettulli $^{1} \cdot$ Antonio Eugenio Chiaravalle $^{1}{ }^{1} \cdot$ Marco lammarino $^{1}$ (i)
}

Received: 19 June 2021 / Accepted: 24 November 2021 / Published online: 27 January 2022

(c) The Author(s) 2021

\begin{abstract}
Strontium-90 (Sr-90) contamination in food is a major public health issue. Several radiochemical methods are available for the determination of Sr-90. However, the application of these procedures is not focused on solid foods, but only on liquid (milk, water, etc.) and environmental matrices, and they were not fully validated. The aims of this work were to establish and validate a fast, sensitive method for the determination of Sr-90 in solid food matrices such as meat and dairy products, seafood, vegetables, and animal feed, using a specific resin for extraction and ultra-low-level liquid scintillation counting for detection. The method was optimised and validated according to relevant legislation. Good analytical performance was obtained, including high specificity and linearity together with low measurement uncertainty (13.1\%). The minimal detectable activity was $11 \mathrm{mBq} \mathrm{kg}^{-1}$, and the mean repeatability (CV\%) and recovery values were $10.7 \%$ and $100.1 \%$, respectively. These parameters assured method applicability for official food safety controls. The method was applied to reference materials and submitted to proficiency test round to confirm its reliability for $\mathrm{Sr}-90$ quantification in solid foodstuffs and feed. The newly established method may be broadly applicable to complex matrices.
\end{abstract}

Keywords Chromatographic extraction $\cdot$ Food safety $\cdot$ Liquid scintillation counting $\cdot$ Radioactive contamination . Strontium-90 · Validation

\section{Introduction}

The discovery of radioactivity in 1896 has led to applications in different sectors, including medicine, nuclear power, and the arms industry. Among radionuclides, Sr-90 is one of the most dangerous fission products with respect to radiotoxicity due to its long physical and biological halflife $(\mathrm{T} 1 / 2=28.50 \mathrm{y})$ (Wilken and Joshi 1991) and chemical similarity to calcium (Vaida and Kim 2010). The similar metabolic behaviour to that of calcium leads to radionuclide accumulation in bone tissues (Iammarino et al. 2018).

Marco Iammarino

marco.iammarino@izspb.it; marco.iammarino@tin.it

1 Chemistry Department, National Reference Center for the Detection of Radioactivity in Feed and Foodstuff, Istituto Zooprofilattico Sperimentale della Puglia e della Basilicata, Foggia, Italy

2 ENEA-Istituto Nazionale di Metrologia delle Radiazioni Ionizzanti, C.R. Casaccia, Rome, Italy
The radioactive decay chain of $\mathrm{Sr}-90$ is as follows:

$\mathrm{Sr}-90 \rightarrow \mathrm{Y}-90 \rightarrow \mathrm{Zr}-90$

where Sr-90 decays in yttrium-90 (Y-90) by emitting a pure beta particle with $E_{\beta, \max }$ of $546 \mathrm{keV}$, while Y-90 decays in stable daughter zirconium-90 (Zr-90), emitting a beta particle with higher energy $\left(E_{\beta, \max }=2280 \mathrm{keV}\right)$. Though Y-90 has a shorter half-life $(\mathrm{T} 1 / 2=64.10 \mathrm{~h})$ than that of Sr-90, it is particularly dangerous because it damages the bones and bone marrow (Cabrera et al. 1999).

After approximately 2 weeks, Y-90 is in secular equilibrium with its parent nuclide; this property is exploited for the indirect determination of Sr-90 activity (dell'Oro et al. 2013). Therefore, the determination of Sr-90 can be performed in two ways: direct or indirect. The first approach involves the direct separation of Sr-90 from the other components and subsequent instrumental measurement. The second approach involves the indirect determination of Sr-90 by the determination of Y- 90 . 
The accidental or deliberate release of radioactive material into the atmosphere leads to the fall-out of radionuclides on water and soil. In Europe, the Chernobyl disaster of 1986 resulted in substantial environmental contamination with $\mathrm{Sr}-90$, involving $200,000 \mathrm{~km}^{2}$ surrounding the accident. Air contamination and rainfall intensity determined the extent of radionuclide deposition on the soil (World Health Organization 2005). The most recent nuclear accident occurred in March 2011 in Fukushima (Japan), where the cooling system failed after a tsunami flooded the power station, causing core reactor meltdown, and resulting in the release of radioactive materials and enormous food safety issues worldwide (Maxwell et al. 2013). Radionuclides may be introduced into the human body by inhalation, which is the most dangerous route of exposure immediately after a nuclear accident, or by ingestion, considered the main route of introduction via the food chain (soil, water vegetation food human). This necessitates food safety controls, especially immediately after a nuclear accident, requiring reliable, robust, and reproducible procedures. A number of methods have been developed for the determination of $\mathrm{Sr}-90$ in the environment and foodstuffs and for calculating transfer factors, following the migration and uptake processes, and/or estimating the dose responses (Grahek et al. 2018).

These methods are based on different techniques, e.g., beta counting (Vaida and Kim 2010; Maxwell et al. 2015), solid or liquid scintillators (Kong et al. 2018), Cerenkov counting (Jiang et al. 2017), and mass spectrometry (Russell et al. 2017). The extraction/separation of $\mathrm{Sr}-90$ from environmental and/or food matrices can be performed in different ways, including the fuming nitric acid method (Livens 1990), liquid-liquid extraction (Happel et al. 2003), ionexchange (Landstetter and Wallner 2006), and extraction chromatography (Jeter and Grob 1994). The majority of these methods are not applicable to food, while the few procedures available, applied in food analysis, have a number of limitations, including the time-consuming processes, use of hazardous reagents, low repeatability and sensitivity, and presence of interfering radionuclides. Moreover, validation procedures are lacking, and this is a strong limitation, since the laboratories in charge of official food controls need fully validated radiochemical procedures in order to fulfil the ISO 17025 requirements.

The aim of this work was to establish and validate a rapid and sensitive method for the determination of Sr-90 activity in food and feed matrices by using specific resin (Sr-Resin) for Sr-90 separation (Surman et al. 2014). The measurement of Sr-90 was obtained by ultra-low-level liquid scintillation counting (LSC). This method was fully validated and then tested on reference materials, various food matrices (meat, fish, vegetables, and cheese), and animal feeds, and submitted to proficiency test round.

\section{Materials and Methods}

\section{Chemicals and Working Standard Solutions}

Nitric acid ( $\geq 65 \% \mathrm{w} / \mathrm{v})$, oxalic acid $(99 \%)$, and aluminium nitrate nonahydrate $(\geq 98.5 \%)$ were supplied by Sigma-Aldrich (Steinheim, Germany). Each sample was treated by using Sr-Resin columns $(10 \mathrm{~mL}$, particle size: $100-150 \mu \mathrm{m}$ ) supplied by Triskem International (Bruz, France). The Ultima Gold AB scintillation cocktail was purchased from PerkinElmer (Waltham, MA, USA). Strontium certified standard solution $\left(10,000 \mathrm{mg} \mathrm{L}^{-1}\right)$ in $4 \%$ nitric acid (HNO3) was purchased from CPI International (Santa Rosa, CA, USA). Sr-90 standardised solution in $0.1 \mathrm{~N} \mathrm{HCl}$ (with $10 \mu \mathrm{g} \mathrm{mL}^{-1}$ of $\mathrm{SrCl}_{2}$ carrier) at a concentration of $3.95 \pm 0.07 \mathrm{kBq} \mathrm{g}^{-1}$ (date: January 1, 2018) was obtained from Physikalisch-Technische Bundesanstalt (Braunschweig, Germany). All solutions were prepared using ultrapure water with a specific resistance of $18.2 \mathrm{M} \Omega$-cm, supplied by a Milli-Q RG unit (Millipore, Bedford, MA, USA).

\section{Analytical Equipment}

Sr-90 activity was detected by using a liquid scintillation counter (Quantulus Wallac 1200; PerkinElmer, Turku, Finland). WinQ Wallac and EASY View Spectrum Analysis Software (PerkinElmer) were used for instrumental control, data acquisition, and elaboration. All acquisition parameters are specified in Table 1.

The Sr "chemical yield" was verified by ICP-MS (ELAN DRC II ICP-Mass Spectrometer (PerkinElmer, Shelton, USA). A stable $\mathrm{Sr}$ calibration curve was obtained by analysing a $\mathrm{Sr}-88$ standard solution in $2 \% \mathrm{HNO}_{3}$ at concentrations of 0.0 (blank), $0.5,2.5,10.0$, and $50.0 \mu \mathrm{g}$ $\mathrm{L}^{-1}$ (internal standard: Rhodium solution $0.2 \mathrm{mg} \mathrm{L}^{-1}$ ). All acquisition parameters used for determination are specified in Table 1 . The strontium yield $\left(Y_{\mathrm{Sr}}\right)$ was calculated by applying the following Eq. (1):

$\mathrm{Y}_{\mathrm{Sr}}=\frac{C_{S r} * V_{1}}{C_{\text {Srcarrier }} * V_{2}} * 100$

where $C_{\mathrm{Sr}}$ is the strontium concentration detected by inductively coupled plasma mass spectrometry (ICP/MS); $V_{1}$ is the volume collected from the column $(150 \mathrm{~mL}) ; C_{\mathrm{Sr} \text { carrier }}$ is the concentration of $\mathrm{Sr}$ carrier added $(1 \mathrm{~mL}$ of $10,000 \mathrm{mg}$ $\mathrm{L}^{-1}$ standard solution in $50 \mathrm{~mL}$, corresponding to $200 \mathrm{mg}$ $\left.\mathrm{L}^{-1}\right)$; and $V_{2}$ is the initial sample volume $(50 \mathrm{~mL})$. 
Table 1 Liquid scintillation counter and inductively coupled mass spectrometer settings

\begin{tabular}{llll}
\hline Liquid scintillation counter (LSC) & & Inductively coupled mass spectrometer (ICP/MS) \\
\hline Coincidence bias & \multirow{2}{*}{ Low } & Radiofrequency generator & $1000-1300 \mathrm{~W}$ \\
& & Gas flow & $15.0 \mathrm{~L} \mathrm{~min}^{-1}$ \\
& \multirow{2}{*}{80} & Argon nebulisation flow & $0.9-1.1 \mathrm{~L} \mathrm{~min}^{-1}$ \\
Pulse shape analysis (PSA) & Pump settings/sample flush & $60 \mathrm{~s}$ \\
& & Sample flush speeding & $-32.0 \mathrm{rpm}$ \\
& & Read delay & $20 \mathrm{~s}$ \\
Channels & \multirow{2}{*}{$300-950$} & Read delay analysis speeding & $-20 \mathrm{rpm}$ \\
& & Wash & $45 \mathrm{~s}$ \\
Acquisition time & Wash speeding & $-32 \mathrm{rpm}$ \\
& \multirow{2}{*}{$480 \mathrm{~min}$} & Scanning settingsdDwell time & $50 \mathrm{~ms}$ \\
& & Sweeps/reading & 20 \\
& Reading/replicate & 1 \\
& & Replicates & 3 \\
& & Scanning mode & Peak hopping \\
\hline
\end{tabular}

\section{Sample Preparation}

As reported by several authors, the radiochemical determination of $\mathrm{Sr}-90$ is a difficult task owing to its chemical affinity with other alkaline earth elements, such as $\mathrm{Ca}$ and $\mathrm{Ba}$. This affinity makes radiochemical separation a long and laborious task. Moreover, the isotopes $\mathrm{Pb}-210$ and Bi-210 may interfere radiometrically with Sr-90 (United States Environmental Protection Agency 2020) making analytical results unreliable. During the last few years, specific resins (extractants) have been developed for the radiochemical separation of several radionuclides from interfering elements present in complex matrices. A resin based on $\left(4,4^{\prime}\left(5^{\prime}\right)\right.$-di- $t$-butylcyclohexano-18-crown-6) (Fig. 1) may be used for the successful extraction of Sr-90 from liquid (i.e., water) and solid matrices (previously mineralised) (Triskem International 2016). However, the application to food samples is limited (Lopes et al. 2010).

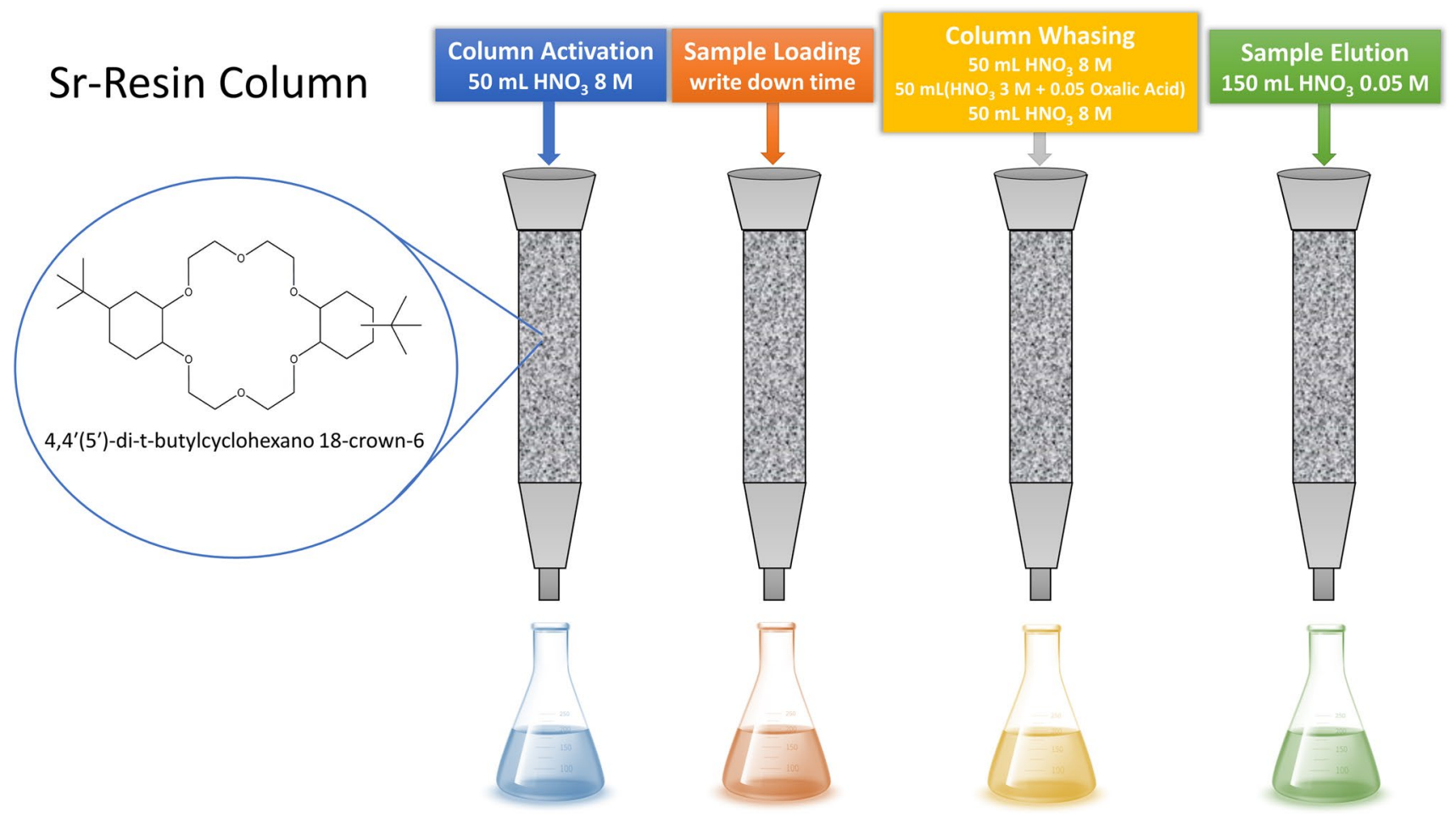

Fig. 1 Optimised sequence for Sr-90 chromatographic extraction by the Sr-Resin column 
This can be attributed to the composition of foodstuffs, rich in minerals that may interfere with separation, making Sr-90 extraction difficult (e.g., in cheese, high calcium content may saturate the active sites of the column). In this study, sample preparation was optimised for the use of specific Sr-Resin columns for food samples analysis. An effective temperature ramp was used to obtain the complete ashing of the sample. Then, different concentrations of $\mathrm{HNO}_{3}(3 \mathrm{M}, 5 \mathrm{M}$, and $8 \mathrm{M})$ and volumes $(25$, 50,75 , and $100 \mathrm{~mL}$ ) were tested for ash solubilisation, accounting for the compatibility with the resin. Based on the literature, different volumes of solutions composed of oxalic acid, nitric acid, and aluminium nitrate were tested for column activation $\left(25,50\right.$, and $100 \mathrm{~mL}$ of $8 \mathrm{M} \mathrm{HNO}_{3}$ ), washing solutions $(25,50$, and $100 \mathrm{~mL})$, and final elution $\left(100,150\right.$, and $200 \mathrm{~mL}$ of $\left.0.05 \mathrm{M} \mathrm{HNO}_{3}\right)$.

\section{Method Optimisation}

Since the pulse shape depends on the vial type and scintillation cocktail/sample characteristics, the discrimination conditions should refer to the specific characteristics of the analysed sample. In this work, a $20-\mathrm{mL}$ polytetrafluoroethylene (PTFE) vial was used. The vial was filled with $8 \mathrm{~mL}$ of $0.1 \mathrm{M} \mathrm{HCl}$ and $12 \mathrm{~mL}$ of Ultima Gold $\mathrm{AB}$ scintillation cocktail, and the optimal value of the pulse shape analysis (PSA) parameter was evaluated by marking two distinct blank samples with $1.5 \mathrm{~Bq}$ of Am-241 $(\mathrm{E}=5.49 \mathrm{MeV})$ and $1.5 \mathrm{~Bq}$ of $\mathrm{Sr}-90 / \mathrm{Y}-90$ solution at secular equilibrium. Subsequently, the percentage of alpha events on the total recorded count in the beta count window and the relative uncertainty (and vice versa) was calculated for each emitter using the following equations:

$\tau_{\alpha}=\frac{\beta}{\alpha+\beta} u_{\tau_{\alpha}}=\frac{\sqrt{\alpha^{2} u_{\beta}^{2}+\beta^{2} u_{\alpha}^{2}}}{(\alpha+\beta)^{2}}$

$\tau_{\beta}=\frac{\alpha}{\alpha+\beta} u_{\tau_{\beta}}=\frac{\sqrt{\alpha^{2} u_{\beta}^{2}+\beta^{2} u_{\alpha}^{2}}}{(\alpha+\beta)^{2}}$

where $\alpha$ is the number of events measured in the alpha window, $\beta$ is the number of events measured in the beta window, and $u_{\alpha}$ and $u_{\beta}$ are the uncertainties in $\alpha$ and $\beta$, respectively, associated with Poisson statistics for radioactive decay.

Finally, the interference parameters $\tau_{\alpha}$ and $\tau_{\beta}$ (Eqs. (2) and (3), respectively) were plotted as a function of the PSA value. The optimal PSA in the present study was 80 , corresponding to alpha interference equal to $0.84 \%$ and beta interference of approximately $0.28 \%$.

\section{Validation Procedure}

Specific guidelines for validating radiochemical methods are not available. As demonstrated previously (dell'Oro et al. 2014; Iammarino et al. 2015), method validation can be carried out following an in-house validation model, developed according to the most representative European regulations and guidelines related to chemical methods (European Commission 2002; Thompson et al. 2002; European Commission 2017; Iammarino 2019). This approach was integrated, where necessary, with specific ISO reference documents, to complete the validation relating to other radiochemical parameters (WHO and FAO 2011).

The Sr-90 counting efficiency $(\varepsilon)$, which is the ratio of the counts per minute (CPM) to disintegrations per minute (DPM), expressed as a percentage (CPM/DPM \%), was calculated by treating the Sr-90 source at four different activity concentrations, $0.1,0.5,1.0$, and $5.0 \mathrm{~Bq} \mathrm{~kg}^{-1}$ (six repetitions each), by the whole sample preparation, as described below (see the "Optimisation of Sample Preparation" section).

Method selectivity is guaranteed by the use of the specific Sr-Resin column, able to separate the radionuclide of interest from other elements and radionuclides that may interfere with the analysis (especially $\mathrm{K}-40$ and $\mathrm{Pb}-214$ ). Selectivity was also evaluated by treating the $\mathrm{Sr}-90$ source in equilibrium with Y-90, collecting the final eluate containing only Sr-90, and verifying the growth of Y-90 by repeated measures.

The limit of quantification (LOQ) and limit of detection (LOD) were assessed using the Currie approach (IAEA 1989; De Felice et al. 2017). As indicated by ISO 11929:2019 (International Organization for Standardization 2019), Part I, §5.6, the decision limit (in $\mathrm{Bq} \mathrm{kg}^{-1}$ ) of a measurand from which a background noise component (or sample blank) is subtracted must be defined by studying the uncertainty associated with the latter. The decision threshold should be chosen to assure that the measurand (with its uncertainty) is significantly higher than the background noise with its uncertainty. The problem is solved, in accordance with Berger (1985), by applying an appropriate decision theory. The decision threshold is defined as:

$P\left(y>y^{*} \mid \tilde{y}=0\right)=\alpha$

That is, the decision threshold is defined by the probability $(\alpha)$ of obtaining a measure (y) greater than the decision threshold $\left(y^{*}\right)$ (where the true value is equal to 0 or not detectable). Using a quadratic loss function, the decision threshold is given by the relationship:

$y^{*}=k_{1-\alpha} * \tilde{u}(0)$

where $k_{1-\alpha}$ represents the (1- $\left.\alpha\right)$ quantile of the normal distribution and $\tilde{u}$ represents the standard uncertainty of the measurand. 
Similarly, the detection limit is defined as the smallest true value for which the probability of an incorrect decision for the presence of the physical effect does not exceed the predefined decision threshold $\beta$ (probability of a false negative):

$P\left(y<y^{*} \mid \tilde{y}=y^{\#}\right)=\beta$

In this case, the decision limit $y^{\#}$ is defined as the smallest of the solutions of the recursive equation:

$y^{\#}=y^{*}+k_{1-\beta} * \tilde{u}\left(y^{\#}\right)$

Generally, the situation in which $\alpha=\beta=0.05$ is considered, and $k_{1-\alpha}=k_{1-\beta}=1.65$.

The linearity of the method was studied by building the calibration curve, using the data obtained from the counting efficiency tests. After obtaining the least squares regression equation, the coefficient of determination and the intercept significance of the calibration curve were evaluated.

The Sr chemical yield was determined by an ICP/MS analysis of six samples composed of ultrapure water (four samples) and animal feed (two samples) fortified with $1 \mathrm{~mL}$ of $\mathrm{Sr}$ carrier $\left(10,000 \mathrm{mg} \mathrm{L}^{-1}\right)$. These samples were treated by the full procedure and then the final $\mathrm{Sr}$ concentration was compared to the initial concentration to calculate the recovery $(\%)$.

Sr-90 activity (A) was evaluated by the following equation:

$A=\frac{\left(r_{g}-r_{0}\right)}{2 * M * f\left(T_{m}\right) * \varepsilon_{S r+Y} * C} e^{\lambda_{S r} T} B q / k g$

where $M$ is the reference mass in kilogram; $\varepsilon_{\mathrm{Sr}+\mathrm{Y}}$ is the counting efficiency of the reference source of Sr-90 at the secular equilibrium with $\mathrm{Y}-90 ; \lambda_{\mathrm{Sr}}$ is the decay constant of $\mathrm{Sr}-90$ in s$^{-1} ; T$ is the time elapsed between the sampling date and the separation date; $r_{\mathrm{g}}$ is the sample count rate $\left(\mathrm{s}^{-1}\right) ; r_{0}$ is the blank count rate $\left(\mathrm{s}^{-1}\right)$; and $C$ is the correction factor for carrier recovery.

The previous relation is derived by integrating the Bateman equations.

Moreover:

$f\left(T_{m}\right)=1-\frac{k_{\varepsilon}}{1+k_{\varepsilon}} * e^{-\lambda_{Y} T_{m}} * \frac{1-e^{-\lambda_{Y} T_{g}}}{\lambda_{Y} T_{g}}$

The equation is a function that measures the growth of Y-90 during the whole measurement period, in which:

$T_{\mathrm{m}}$-is the time interval between chromatographic separation and the start of counting

$\lambda_{Y}$-is the decay constant of Y-90 in s ${ }^{-1}$

$\mathrm{T}_{\mathrm{g}}$-is the counting time (s) $\mathrm{k}_{\varepsilon}$-is the ratio of the counting efficiencies of a pure Y-90 emitter and a pure Sr-90 emitter, considering that the two sources have the same geometry

The counting efficiency is a measure of the fraction of events producing a measurable pulse. Therefore, it depends not only on the scintillation efficiency (i.e., the average number of photons produced per unit of energy), but also on the photomultiplier characteristics and on the properties of the electronic measuring instrument. The total counting efficiency was evaluated by measuring a source of Sr-90/Y-90 at an activity concentration of $0.1,0.5,1.0$, and $5.0 \mathrm{~Bq} \mathrm{~kg}^{-1}$. This efficiency is routinely used in the evaluation of the Sr-90 activity concentration. It differs significantly from the sum of the separate efficiencies for the single radionuclide Sr-90 and Y-90.

The $k$ parameter contributes to the evaluation of the Y-90 growth factor both during the time between elution and instrumental reading and during the reading itself. The factor was evaluated by reading separately the two sources of Sr-90 and Y-90. The first was prepared by spiking ultrapure water with a certified solution of Sr-90/Y-90 followed by the specified technical procedure, and the second was prepared using the washing solution in which Y-90 is present. Ten values were obtained for each source, correcting for Y-90 ingrowth/decay.

The method accuracy was verified by estimating precision (as CV\%) and trueness (as recovery \%). A sample of animal feeds was prepared ad hoc for this validation step. The choice of an appropriate and representative matrix for evaluating method accuracy was based on two main requirements: (i) matrix composition; (ii) matrix contamination by Sr-90. More in depth, in order to simulate the most complex analytical conditions, a matrix characterised by both high levels of possible interfering metals and detectable amounts of Sr-90 was chosen. Since the composition and possible Sr-90 contamination is highly variable among different types of feed (Iammarino et al. 2015), a "mix" of several kinds of feed was prepared. Portions (175 g each) of forage, corn, bran, and complementary feed were ashed and carefully homogenised. This "pool" of animal feed ashes was subdivided in portions corresponding to $50.0 \mathrm{~g}$ of fresh weight. Two samples were analysed to evaluate the activity concentration of Sr-90 (if present). The remaining 12 portions were fortified at two levels $\left(0.5 \mathrm{~Bq} \mathrm{~kg}^{-1}\right.$ and $1.0 \mathrm{~Bq} \mathrm{~kg}^{-1}$, six repetitions each) and analysed.

Ruggedness under major changes condition was evaluated using different matrix types (two repetitions for each type). The recovery $\%$ and $\mathrm{CV} \%$ were compared with those obtained during the validation using animal feeds. The following samples were utilised (50 g each): cheese pool, including hard cheese, spreadable fresh cheese, and slices of sweet cheese; meat pool, including hamburger, fresh 
sausage, and mortadella; fish pool, including sea bream, sea bass, mussels, clams, prawns, and cuttlefish; and vegetable pool (frozen vegetable soup). Each sample was analysed before fortification in duplicate to ensure the absence of $\mathrm{Sr}-90$ (<LOQ).

The measurement uncertainty was calculated according to UNI CEI ENV 13,005:2000 (Ente Italiano di Normazione 2000) and ISO 21748:2010 (International Organization for Standardization 2010). The following sources of uncertainty were evaluated and included in the final calculation: net counting, sample weight, $\mathrm{Sr}$ chemical yield, counting efficiency, Y-90 decay time, and repeatability.

\section{Proficiency Test Round}

In May 2021, this radioanalytical method was tested within a Proficiency Test organised by ERA (Waters ${ }^{\mathrm{TM}}$, Golden, CO, USA, an accredited ISO/IEC 17,043:2010 agency), involving 32 laboratories from different countries. A sample composed of a mix of different vegetables was analysed for the quantification of $\mathrm{Sr}-90$. The measured value was $36.7 \pm 4.8 \mathrm{~Bq} \mathrm{~kg}^{-1}$, against a true value of $43.5 \mathrm{~Bq} \mathrm{~kg}^{-1}$ (satisfactory range: $31.2-55.9 \mathrm{~Bq} \mathrm{~kg}^{-1}$ ), resulting in a Z-score equal to -1.11 (Table 3 ).

\section{Results and Discussion}

\section{Optimisation of Sample Preparation}

The proposed method includes three steps: sample ashing, Sr-90 separation by a Sr-Resin column, and determination of Sr-90 activity by LSC. The first two steps were optimised with respect to ashing times and temperatures (to minimise the times and obtain clear ashes without residues) and $\mathrm{Sr}$ Resin column usage was optimised. The optimised sample preparation procedure was as follows: $50 \mathrm{~g}$ of homogenised sample was ashed using the following temperature ramp, $250{ }^{\circ} \mathrm{C}(5 \mathrm{~h}), 550{ }^{\circ} \mathrm{C}(14 \mathrm{~h}), 600{ }^{\circ} \mathrm{C}(5 \mathrm{~h})$. This procedure is faster than the conventional approach (Iammarino et al. 2017), which requires almost 3 days for completion. This improvement was achieved by using containers composed of more resistant material (zirconium) in place of porcelain. After cooling, the ashes were dissolved in $50 \mathrm{~mL}$ of $65 \%(\mathrm{w} / \mathrm{v}) \mathrm{HNO}_{3}$, directly in the container, transferred in a 500 -mL glass flask, and $1 \mathrm{~mL}$ of $10,000 \mathrm{mg} \mathrm{L}^{-1}$ strontium standard solution ( $\mathrm{Sr}$ carrier) was added. Then, a first aliquot of $50 \mu \mathrm{L}$ of the solution was withdrawn, transferred to a $50-\mathrm{mL}$ volumetric flask, and diluted to $50 \mathrm{~mL}$ by the addition of $0.1 \mathrm{M} \mathrm{HNO}_{3}$. This step is used for determining the $\mathrm{Sr}$ chemical yield by inductively coupled plasma mass spectrometry (ICP/MS). The traditional procedure used for chemical yield verification, performed by weighing the final counting form of the element, as a precipitate recovered on filter papers or on counting discs composed of inert metals, is based on a series of analytical steps that may compromise method reproducibility. Inversely, ICP/MS guarantees high sensitivity, selectivity, and accuracy (Iammarino et al. 2016a, 2015). If necessary, the mixture was treated by leaching at $320^{\circ} \mathrm{C}$ on a hot plate for a few minutes to completely solubilise the ashes. The mixture was then filtered under a vacuum $(\sim 0.3 \mathrm{bar})$ using Purabind 0.45 filters $(0.45 \mu \mathrm{m}$, $47 \mathrm{~mm}$; Whatman, Dassel, Germany) and $25 \mathrm{~mL}$ of $2 \mathrm{M}$ aluminium nitrate was added. A Sr-Resin column was placed on a vacuum chamber (a maximum vacuum of 0.3 bar may be applied, if necessary) and $\mathrm{Sr}-90$ extraction was performed following the optimised scheme presented in Fig. 1. Indeed, the complete elution of stable $\mathrm{Sr}$ was achieved using $150 \mathrm{~mL}$ of $0.05 \mathrm{M} \mathrm{HNO}_{3}$. This result was confirmed by the experimental construction of the elution curve using stable Sr. A second aliquot of $50 \mu \mathrm{L}$ of the final solution eluted from the column was withdrawn, transferred into a $50-\mathrm{mL}$ volumetric flask, and diluted to $50 \mathrm{~mL}$ by the addition of $0.1 \mathrm{M} \mathrm{HNO}_{3}$ for subsequent Sr-90 chemical yield determination by ICP/ MS, comparing the mass concentrations of the two withdrawn aliquots. The solution was then evaporated to dryness on a hot plate; the residue was dissolved in $8 \mathrm{~mL}$ of $0.05 \mathrm{M}$ $\mathrm{HNO}_{3}$ (to avoid any loss of counting efficiency due to the high quenching effect of high molar $\mathrm{HNO}_{3}$ solutions) and transferred in a scintillation vial, together with $12 \mathrm{~mL}$ of scintillation cocktail. This mixture was used for the determination of Sr-90 by ultra-low-level LSC. Counting was started $2 \mathrm{~h}$ after the addition of the scintillation cocktail, since this time gap ensures the resetting of both chemiluminescence and photoluminescence.

\section{Optimisation of Measurement Conditions}

The optimal alpha-beta discrimination conditions can be identified by the PSA parameter value such that the PSA circuit better discriminates alpha from beta events. Two samples were measured at different PSA, one traced with a pure alpha emitter and the other traced with a pure beta emitter, to evaluate the percentage of events recorded in the wrong counting window. The optimal operating condition corresponds to the situation in which both alpha interference (\% of alpha events recorded in the beta counting window) and beta interference ( $\%$ of beta events recorded in the alpha counting window) are minimised. The optimal condition corresponds to the intersection point of two curves. The optimised acquisition parameters of both LSC and ICP-MS are reported in Table 1. The detector channels used for spectral acquisition were selected to exclude the counting rate due to photoluminescence and emission of $\mathrm{H}-3$ (which may be present in both reagents and samples). In these conditions, the total efficiency was $0.996 \pm 0.036$. The efficiencies of 
Sr-90 and Y-90 were 0.51 and 0.61 , respectively, with a calculated $k$ factor of 1.19 .

\section{Validation Parameters}

Critical validation parameters are summarised in Table 2.

Selectivity The selectivity of a method represents the ability to determine the analyte of interest in the presence of interference. Selectivity was conferred by the specific Sr-resin chromatographic column capable of separating the analyte of interest from matrix interferences (e.g., Ca, K-40, and $\mathrm{Pb} / \mathrm{Bi}$ isotopes).

A separation test of a $\mathrm{Sr}-90$ source in secular equilibrium with Y-90 was carried out to evaluate the selectively of the chromatographic column. A feed sample with a high K-40 content (about $750 \mathrm{~Bq}$, measured by HPGe gamma spectrometry) was fortified with this source, and the ingrowth of Y-90 in the final eluate containing only Sr-90 and the decay of Y-90 in the waste were evaluated. A series of 15 successive readings excluded the presence of potassium in the final eluate and confirmed the presence of a strong signal from K-40 in the waste. In the latter case, as expected, the final CPM values were in agreement with the blank value of $1.840 \pm 0.096$ CPM (Fig. 2). The selectivity of the method was confirmed following the growth of Y-90 obtained from repeated measures on the final eluate containing only $\mathrm{Sr}-90$ (Fig. 3A).

Linearity The linearity of the method was studied by building a calibration curve using the levels adopted during the counting efficiency tests $\left(0.1,0.5,1.0\right.$, and $\left.5.0 \mathrm{~Bq} \mathrm{~kg}^{-1}\right)$. The coefficient of determination $\left(r^{2}\right)$ was 0.9995 , confirming linearity within the range: LOQ $(0.024$, see the "Characteristic
Limits" section) to $5.0 \mathrm{~Bq} \mathrm{~kg}^{-1}$. Obviously, the measurement range may be expanded by opportune sample dilution.

Sr Chemical Yield The Sr chemical yield was assessed by ICP/MS using six samples (four water and two feed) fortified with $1 \mathrm{~mL}$ of $\mathrm{Sr}$ carrier. The yield was $89.2 \pm 11.8 \%$.

Accuracy The accuracy of the method was evaluated by analysing the validation matrix (see the "Validation Procedure" section) fortified at two levels $\left(0.5\right.$ and $1.0 \mathrm{~Bq} \mathrm{~kg}^{-1}$, 6 repetitions each). The recovery $\%$ and $\mathrm{CV} \%$ values are reported in Table 2 . The mean recovery percentage was $100.1 \%$ and the CV\% was less than $13.1 \%$. These parameters are comparable to those of other methods (Iammarino et al. 2016b), supporting the accuracy of the method. Accuracy was also evaluated by five replicated measurements of the IAEA-154 certified matrix (whey powder), whose recommended value on August 31, 1987, was $6.90 \mathrm{~Bq} \mathrm{~kg}^{-1}$ (95\% confidence interval: 6.00-8.00), and an average value of $6.14 \pm 0.80 \mathrm{~Bq} \mathrm{~kg}^{-1}$ was obtained. The good agreement with the declared value for the reference material (Z-score: 0.69, En-score: 0.47 ) confirmed the accuracy of the method. Figure 3B shows three representative spectra for samples analysed during validation (Table 3 ).

Robustness Briefly, robustness refers to the ability of the method to yield consistent results, irrespective of variation introduced during its execution. Robustness was evaluated under major changes condition on alternative matrices: cheese, meat, fish, and vegetables. Each alternative matrix was formulated by pooling various products (see the "Validation Procedure" section).

Each pooled sample was first analysed to verify the absence of Sr-90 (activity concentration lower than the decision threshold). Following this verification, each pooled

Table 2 Validation parameters

\begin{tabular}{ll}
\hline Validation parameter & Fortification level $\left(\mathrm{Bq} \mathrm{kg}^{-1}\right)$ \\
\cline { 2 - 2 } & 0.50 \\
\hline Mean value measured $\left(\mathrm{Bq} \mathrm{kg}^{-1}\right)(\mathrm{n}=6) \pm \mathrm{SD}$ & $0.55 \pm 0.05$ \\
$\mathrm{CV} \%(\mathrm{n}=6)$ & 8.3 \\
Mean recovery $(\mathrm{n}=6)$ & $109.7 \%$ \\
Decision threshold $\left(\mathrm{Bq} \mathrm{kg}^{-1}\right)(\mathrm{LOD})$ & 0.011 \\
Detection limit $\left.(\mathrm{Bq} \mathrm{kg})^{-1}\right)(\mathrm{LOQ})$ & 0.024 \\
Sr chemical yield & $89.2 \%$ \\
Linearity $\left(\mathrm{r}^{2}\right)$ & 0.9995 \\
Selectivity & No Interfering \\
Counting efficiency $(\varepsilon) \pm \mathrm{SD}$ & $0.996 \pm 0.12$ \\
Measurement uncertainty & $13.1 \%$ \\
Ruggedness-major changes $(\mathrm{CV} \%)$ & Meats $(0.1 \%)$, dairy products $(10.0 \%)$, seafood (10.0\%), vegetables $(2.0 \%)$, \\
& animal feed (validation matrix) \\
\hline
\end{tabular}




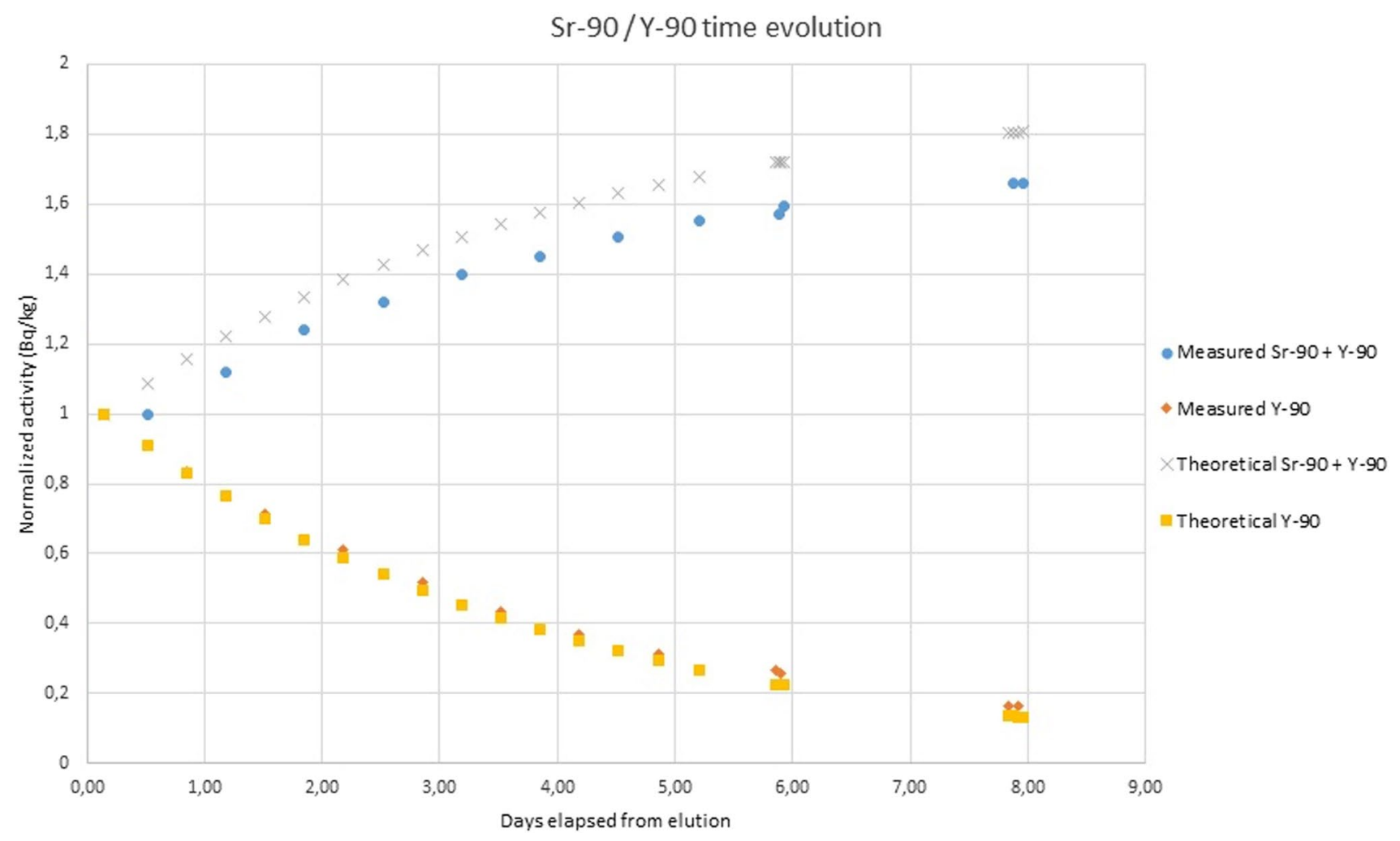

Fig. 2 Time evolution of measured Sr-90 (alone) and Y-90 (alone) sources and the theoretical trend

sample was spiked with $\mathrm{Sr}-90$ at an activity concentration of $0.5 \mathrm{~Bq} \mathrm{~kg}^{-1}$. The resulting samples were processed according to the method described above. CV\% values for each pool, as an indicator of robustness, were lower than $10.0 \%$ (Table 2). This result supports the broad application of the method to solid food matrices (cheese, meat, seafood, and vegetables) and animal feed, since CV\% calculated were lower than the estimated value obtained during validation (10.7\%). Moreover, considering the ductility of the Sr-Resin, it is possible that the method can also be applied to environmental solid matrices, such as soil and solid waste, in addition to food liquid matrices (e.g., milk), after proper sample solidification before ashing.

Characteristic Limits To evaluate the characteristic limits, a Bayesian approach was used.

By replacing the quantities $\tilde{u}(y)$ with the uncertainties determined during the validation and solving the associated equations recursively, the following numerical results were obtained (10) and (11):

$L O D=y^{*}=0.011 \mathrm{Bqkg}^{-1}$

$L O Q=y^{\#}=0.024 B q g^{-1}$
The sensitivity of this procedure was higher than those of various previously established approaches for the determination of Sr-90 in solid matrices (Heilgeist 2000; Kabai et al. 2011; Shuzhong et al. 2015; Temba et al. 2011) and comparable to several others (Gerstmann and Tschöpp 2006; Lopes et al. 2010; Suomela et al. 1993).

Assessment of Measurement Uncertainty All sources of uncertainty with substantial contributions to overall uncertainty were evaluated, as proposed by the UNI CEI ENV 13,005:2000 standard and in accordance with the ISO 21748:2010 standard, which accounts for repeatability. The relative contributions of sources uncertainties were as follows: net counts $(2.80 \%)$, sample mass $(0.01 \%)$, strontium yield (11.2\%), counting efficiency (3.7\%), ingrowth of Y-90 $(3.0 \%)$, and repeatability (3.9\%). Thus, the overall measurement uncertainty was $13.1 \%$.

Owing to the established selectivity of the method, its extension to proportional counting is straightforward, given the lower background noise (counting efficiency generally lower than that for the liquid scintillator) and suitability for routine controls. In fact, the sample in the final reduction step can be dried directly on a stainless-steel counting planchet and checked for Sr-90. In this case, the sensitivity 
A

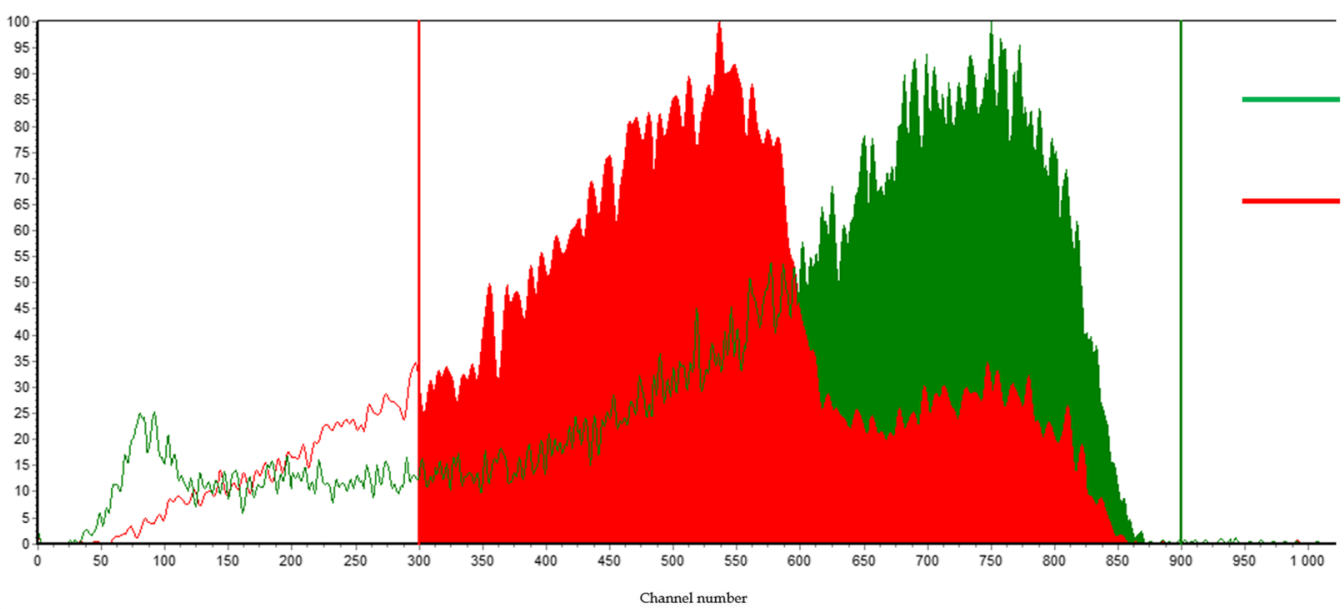

B

Channel number

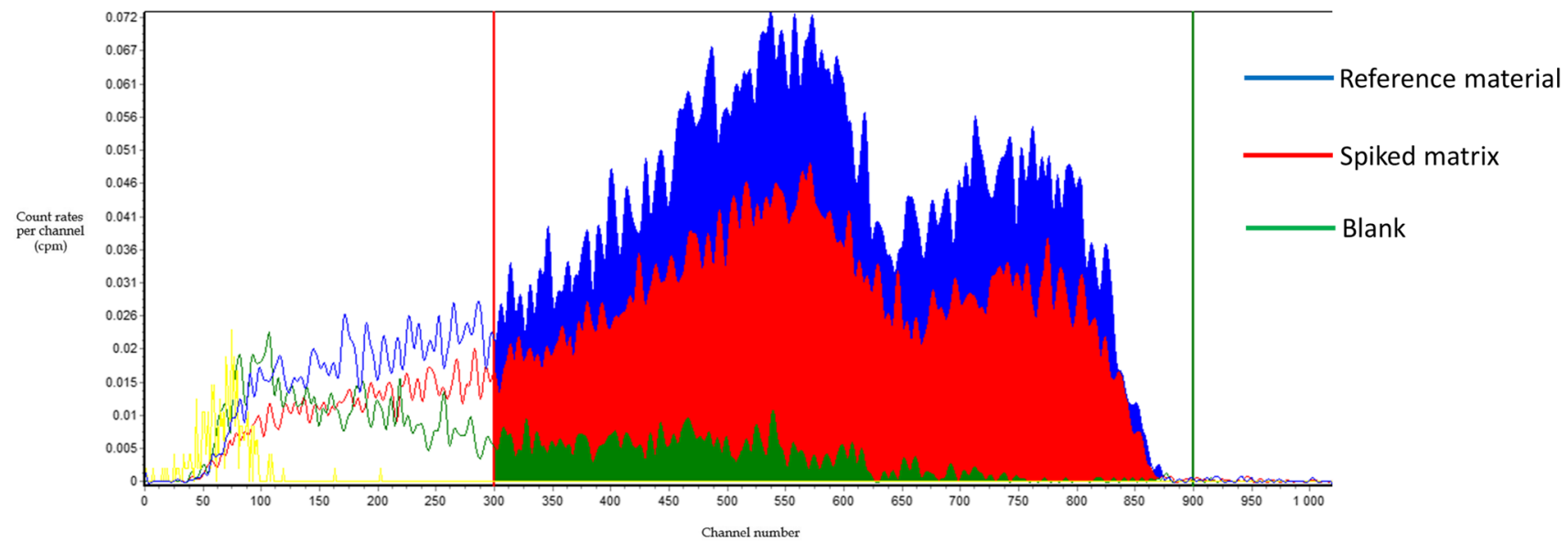

Fig. 3 Spectral overlay: A pure Sr-90 source (red) and Y-90 growth over 15 days (green); B three samples, IAEA-154 (blue), spiked feed (red), and blank sample (green)

Table 3 Proficiency test and reference material analysis

\begin{tabular}{|c|c|c|c|}
\hline Validation session & $\begin{array}{l}\text { Sr-90 activity concentration }(\mathrm{Bq} \\
\left.\mathrm{kg}^{-1}\right) \pm \text { measurement uncertainty }\end{array}$ & $\begin{array}{l}\text { Reference value }(\mathrm{Bq} \\
\left.\mathrm{kg}^{-1}\right) \pm \text { uncertainty }(2 \sigma)\end{array}$ & $\begin{array}{l}\text { Acceptability } \\
\text { range }(\mathrm{Bq} \\
\left.\mathrm{kg}^{-1}\right)\end{array}$ \\
\hline Reference material & $6.1 \pm 0.8$ & $6.9 \pm 1.1$ & $5.8-8.0$ \\
\hline Proficiency test & $36.7 \pm 4.8$ & $43.5 \pm 12.3$ & $31.2-55.8$ \\
\hline
\end{tabular}

should be slightly lower, since proportional counting is less efficient than LSC.

\section{Proficiency Test Round}

The sample of vegetables analysed within the Proficiency test gave a Sr-90 activity concentration equal to $31.1 \mathrm{~Bq} \mathrm{~kg}^{-1}$. The Z-score value obtained was satisfactory, confirming the accuracy of this radioanalytical method for the quantitative determination of $\mathrm{Sr}-90$ in solid foods.

\section{Application to Commercial Samples}

During validation, several solid food samples were collected from local stores (stored in the laboratory at $-18^{\circ} \mathrm{C}$ ) and analysed to verify the applicability of the method to routine radio-surveillance in food safety. Pooled samples of solid foods were prepared. The $\mathrm{Sr}-90$ activity concentrations were lower than the LOQ $\left(11.0 \mathrm{mBq} \mathrm{kg}^{-1}\right)$, confirming the negligible level of Sr-90 in solid foods. Several animal feed samples were also analysed during official radio-surveillance activity at Italian National Reference Center for 
the Detection of Radioactivity in Feed and Foodstuffs. The Sr-90 activity concentrations detected, in the range of LOQ to $4.22 \mathrm{~Bq} \mathrm{~kg}^{-1}$, are reported in Table 3 . The mean activity concentrations of Sr-90 detected in animal feeds were comparable to those reported by Iammarino et al. (2015) which registered mean levels equal to $0.77,2.07$, and $2.93 \mathrm{~Bq} \mathrm{~kg}^{-1}$ in animal feed, silage, and hay, respectively. This brief monitoring confirmed that animal feeds (especially not processed feed, such as hay) might be contaminated by not negligible levels of Sr-90. Another confirmation of this important aspect of food safety and animal wellness is the low percentage of samples with not quantifiable activity concentrations of Sr-90 (only 1 sample out of 24 analysed, $0.04 \%$ ). So, the application of this novel radiochemical approach for the determination of Sr-90 in solid matrices confirmed its reliability, particularly for the quantification of this radionuclide in animal feed.

\section{Comparison to Other Available Methods}

The proposed radiochemical method presents several aspects of novelty, representing an improvement from the current state-of-the-art. Indeed, some rapid radiochemical procedures for the determination of Sr-90, which use extraction chromatographic resins, are already available in the literature (Maxwell et al. 2010; 2016; Maxwell 2018). However, these methods were applied to other types of matrix than food (i.e., limestone, marble, vegetation, etc.). In food analysis, method selectivity is one of the most significant parameters to ascertain during validation, since it is high variable among different foods, and it must be assured for avoiding "false positive" responses. The main effort of this study was the ascertainment of method applicability to the highest number of wide-consumed foods, such as meat, cheese, seafood, and vegetables, through the fundamental verification of validation parameters such as selectivity and accuracy.

The main advantage of this method over other methods used for foodstuffs analysis (Lopes et al. 2010; Iammarino et al. 2016a) is the ability to obtain fast and accurate results without waiting for Y-90 ingrowth. Thus, the method enables the final quantification of $\mathrm{Sr}-90$ in $48 \mathrm{~h}$ (excluding the ashing step), while the Y-90 analysis requires more than 15 days to reach secular equilibrium. As a consequence, the times and cost are significantly reduced. Comprehensive validation, with analyses of all radiochemical parameters, was performed in this study. Lopes et al. (2010) reported some relevant parameters, such as the $\mathrm{CV} \%$ and recovery percentage. However, only three repetitions were performed
Table 4 Evaluation of Sr-90 contamination in different types of animal feed

\begin{tabular}{|c|c|c|c|}
\hline Matrix & $\begin{array}{l}\text { Measured } \mathrm{Sr}-90 \text { activity concen- } \\
\text { tration }\left(\mathrm{Bq} \mathrm{kg}{ }^{-1}\right)\end{array}$ & $\begin{array}{l}\text { Uncertainty }(\mathrm{Bq} \\
\left.\mathrm{kg}^{-1}\right)\end{array}$ & $\begin{array}{l}\text { Feed type mean } \mathrm{Sr}-90 \\
\text { activity concentration }(\mathrm{Bq} \\
\left.\mathrm{kg}^{-1}\right)\end{array}$ \\
\hline Hay & 1.56 & 0.25 & 2.45 \\
\hline Hay & 2.49 & 0.40 & \\
\hline Hay & 2.35 & 0.38 & \\
\hline Hay & 4.22 & 0.68 & \\
\hline Hay & 1.64 & 0.26 & \\
\hline Wheat & 0.76 & 0.12 & 0.39 \\
\hline Wheat & 0.32 & 0.05 & \\
\hline Wheat & 0.09 & 0.01 & \\
\hline Animal feed & 0.13 & 0.02 & 0.26 \\
\hline Animal feed & 0.25 & 0.04 & \\
\hline Animal feed & 0.10 & 0.02 & \\
\hline Animal feed & 0.57 & 0.09 & \\
\hline Animal feed & 0.32 & 0.05 & \\
\hline Animal feed & 0.21 & 0.03 & \\
\hline Beet & 0.09 & 0.01 & 0.09 \\
\hline Beet & $<\operatorname{LOQ}(0.024)$ & - & \\
\hline Oat & 0.75 & 0.12 & 0.83 \\
\hline Oat & 0.44 & 0.07 & \\
\hline Oat & 0.79 & 0.13 & \\
\hline Oat & 1.34 & 0.21 & \\
\hline Corn & 0.22 & 0.04 & 0.31 \\
\hline Corn & 0.31 & 0.05 & \\
\hline Corn & 0.26 & 0.04 & \\
\hline Corn & 0.45 & 0.07 & \\
\hline
\end{tabular}




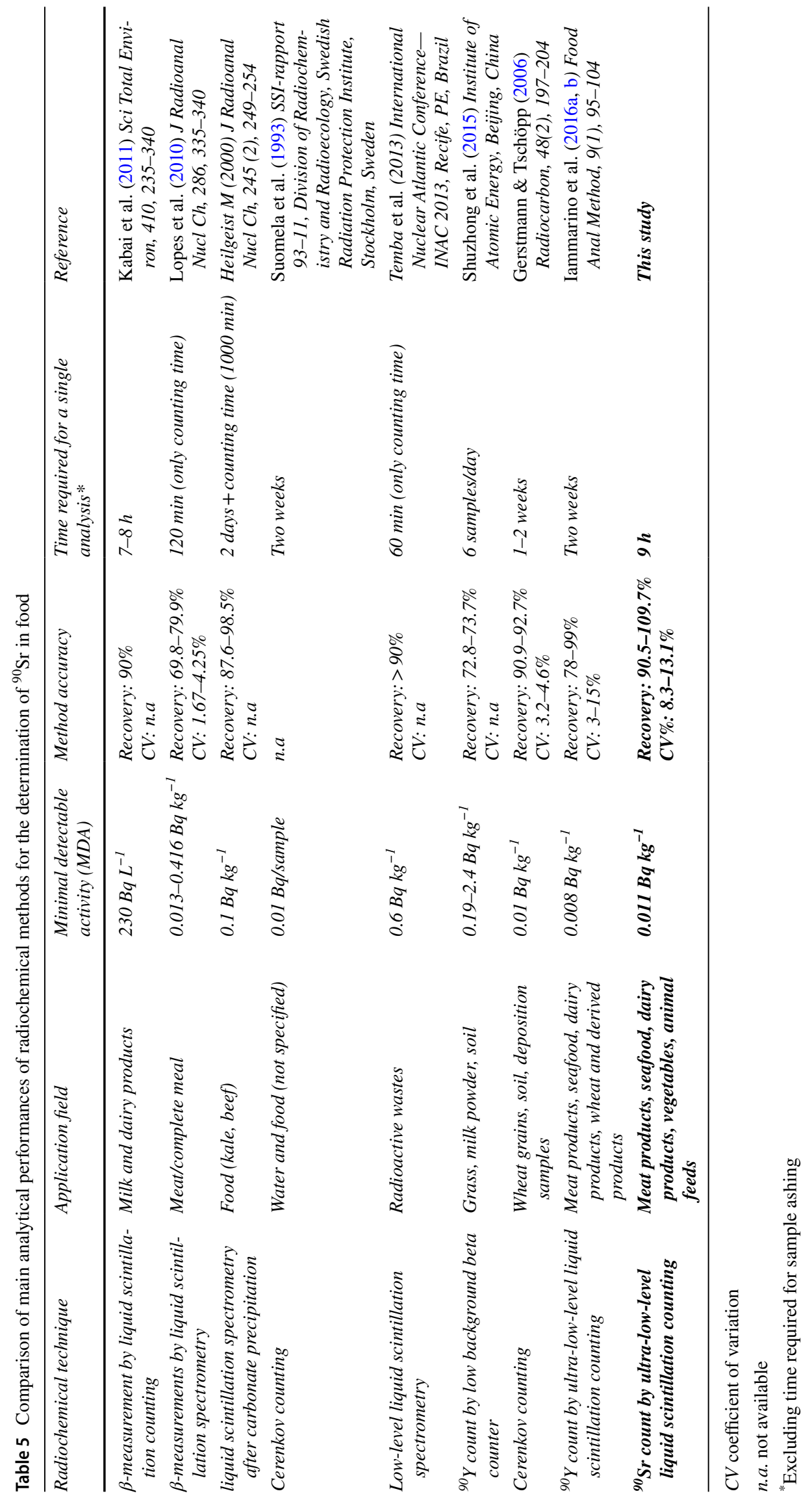


for each level, using very high concentration activities, not common in the routine control of food and feed. Compared to the validation parameters reported by Iammarino et al. (2016a), the CV\% is similar, the mean recovery is higher, and the measurement uncertainty is slightly lower for the newly proposed method. For samples of equal weights, the LOD and LOQ values are lower. Accordingly, the present method is more sensitive due to the higher counting efficiency and chemical recovery, even compared with the method published by Pan et al. (2017). Using this method, it is important to control the precise measurement of the time between Sr-90 elution and instrumental counting. In fact, in this method, the determination of the contribution of Y-90 is crucial for the precise quantification of the Sr-90 activity concentration.

In Table 4, the comparison of main analytical performances of radiochemical methods for the determination of ${ }^{90} \mathrm{Sr}$ in food, actually available, is reported. From this table, the main advantages of the proposed method are well appreciable. The most important is the application field, which allows applying the procedure for all most important solid matrices exploiting, as stated above, the ductility of the $\mathrm{Sr}$ Resin. If compared to the only method comparable under this point of view (Iammarino et al. 2016a), there is an evident advantage in terms of time requested for a single analysis. Another substantial novelty of this study is the proposed approach for the development of a full validation procedure in radiochemical determinations. This approach allows the verification of most important analytical parameters and of measurement uncertainty that is a rarely estimated parameter within this context Table 5.

\section{Conclusion}

The proposed method for the determination of Sr-90 in solid foodstuffs is very simple and suitable for routine analyses of food samples. The method is based on Sr-90 extraction/ separation by specific $\mathrm{Sr}$-Resin and detection/quantification by ultra-low-level LSC. If compared to others available, this approach is more manageable in terms of times, costs, operator safety, reagents consumption, and waste. The method was optimised and validated following an in-house validation model according to relevant legislation. Good analytical performance was obtained in terms of specificity, linearity, sensitivity (minimal detectable activity: $11.0 \mathrm{mBq} \mathrm{kg}{ }^{-1}$ ), mean repeatability (CV\%: 10.7\%), mean recovery $(89.2 \%)$, and measurement uncertainty (13.1\%). The procedure was also applied to reference materials and commercial samples, confirming its reliability. All these verifications assured method applicability in routinely control activity of Sr-90 levels in solid foods and animal feed. Finally, the successful application of the procedure to highly complex matrices, like feed and forages, suggests that it can be extended to environmental samples, like soil and waste, even in the case of nuclear emergencies.

Author Contribution Giuliana Marchesani-validation, conceptualisation, data curation, writing.

Gabriele Trotta-validation, writing, resources, software.

Pierino De Felice—supervision, visualisation.

Nicola Bortone-methodology, resources.

Rita Damiano-resources, validation.

Michele Nicolini-resources, validation.

Rosario Accettulli-resources.

Antonio Eugenio Chiaravalle-project administration, funding acquisition, supervision, visualisation.

Marco Iammarino - conceptualisation, methodology, writing, funding acquisition.

Funding This work was supported by Ministero della Salute (Rome, Italy), who financed the projects GR-2013-02358862 and IZS PB 03/18 RC.

Data Availability The datasets generated during and/or analysed during the current study are available from the corresponding author on reasonable request.

\section{Declarations}

Ethics Approval Not applicable.

Informed Consent Not applicable.

Conflict of Interest Giuliana Marchesani, Gabriele Trotta, Pierino De Felice, Nicola Bortone, Rita Damiano, Michele Nicolini, Rosario Accettulli, Antonio Eugenio Chiaravalle, and Marco Iammarino declare that they have no conflict of interest regarding the publication of this paper.

Open Access This article is licensed under a Creative Commons Attribution 4.0 International License, which permits use, sharing, adaptation, distribution and reproduction in any medium or format, as long as you give appropriate credit to the original author(s) and the source, provide a link to the Creative Commons licence, and indicate if changes were made. The images or other third party material in this article are included in the article's Creative Commons licence, unless indicated otherwise in a credit line to the material. If material is not included in the article's Creative Commons licence and your intended use is not permitted by statutory regulation or exceeds the permitted use, you will need to obtain permission directly from the copyright holder. To view a copy of this licence, visit http://creativecommons.org/licenses/by/4.0/.

\section{References}

Berger JO (1985) Statistical decision theory and bayesian analysis, 2nd edn. Springer, Berlin

Cabrera WE, Schrooten I, Broe MED, D'Haese PC (1999) Strontium and bone. J Bone Miner Res 14(5):661-668. https://doi.org/10. 1359/jbmr.1999.14.5.661 
De Felice P, Jerome S, Petrucci A (2017) Practical implementation of ISO 11929:2010. Appl Radiat Isotopes 126:256-262. https://doi. org/10.1016/j.apradiso.2017.02.004

dell'Oro D, Iammarino M, Bortone N, Chiaravalle AE (2013) Survey on the presence of ${ }^{90} \mathrm{Sr}$ in milk samples by a validated ultra low level liquid scintillation counting (LSC) method. E3S Web of Conferences 1:39005. https://doi.org/10.1051/e3sconf/20130 139005

dell'Oro D, Iammarino M, Bortone N, Mangiacotti M, Chiaravalle AE (2014) Determination of radiostrontium in milk samples by ultra-low-level liquid scintillation counting: a validated approach. Food Addit Contam A 31(12):2014-2021. https://doi.org/10.1080/ 19440049.2014 .968883

Ente Italiano di Normazione (2000) UNI CEI ENV 13005 - Guide to the expression of uncertainty in measurement.

European Commission (2002) Commission Decision 2002/657/EC of 12 August 2002 implementing Council Directive 96/23/EC concerning the performance of analytical methods and the interpretation of results. Official Journal of the European Communities 45(L 221):8-36.

European Commission (2017) Commission Regulation (EU) 2017/625 of the European Parliament and of the Council of 15 March 2017. Off J Eur Union L95:1-142

Gerstmann UC, Tschöpp V (2006) Determination of ${ }^{90} \mathrm{Sr} /{ }^{90} \mathrm{Y}$ in wheat grains, soil, and deposition samples by TBP extraction and Cerenkov counting. Radiocarbon 48(2):197-204. https://doi.org/10. 1017/S003382220006639X

Grahek Ž, Dulanská S, Karanović G, Coha I, Tucaković I, Nodilo M et al (2018) Comparison of different methodologies for the ${ }^{90} \mathrm{Sr}$ determination in environmental samples. J Environ Radioactiv 181:18-31. https://doi.org/10.1016/j.jenvrad.2017.10.012

Happel S, Streng R, Vater P, Ensinger W (2003) Sr/Y separation by supported liquid membranes based on nuclear track micro filters. Radiat Meas 36(1):761-766. https://doi.org/10.1016/S13504487(03)00242-7

Heilgeist M (2000) Use of extraction chromatography, ion chromatography and liquid scintillation spectrometry for rapid determination of strontium-89 and strontium-90 in food in cases of increased release of radionuclides. J Radioanal Nucl Ch 245:249-254. ttps:// akademiai.com/doi/abs/10.1023/A\%3A1006777416037

Iammarino M (2019) Simplified guidelines for chromatographic methods validation. LAP Lambert Academic Publishing, Republic of Moldova

Iammarino M, dell'Oro D, Bortone N, Chiaravalle AE (2015) Beta emitter radionuclides $\left({ }^{90} \mathrm{Sr}\right)$ contamination in animal feed: validation and application of a radiochemical method by ultra low level liquid scintillation counting. Ital J Food Saf 4:4531. https://doi. org/10.4081/ijfs.2015.4531

Iammarino M, dell'Oro D, Bortone N, Mangiacotti M, Chiaravalle AE (2016a) Optimisation and validation of a multi-matrix ultrasensible radiochemical method for the determination of radiostrontium in solid foodstuffs by liquid scintillation counting. Food Anal Method 9(1):95-104. https://doi.org/10.1007/s12161-015-0182-y

Iammarino M, dell' Oro D, Bortone N, Mangiacotti M, Chiaravalle AE (2018) Radiostrontium accumulation in animal bones: development of a radiochemical method by ultra low-level liquid scintillation counting for its quantification. Vet Ital 54(1):41-47

Iammarino M, dell'Oro D, Bortone N, Mangiacotti M, Damiano R, Chiaravalle AE (2016b) Radiostrontium levels in foodstuffs: 4-years control activity by Italian reference centre, as a contribution to risk assessment. Food Chem 210:344-354. https://doi.org/ 10.1016/j.foodchem.2016.04.118

Iammarino M, Mangiacotti M, Miedico O, Bortone N, Damiano R, Chiaravalle AE (2017) Assessment of beta-emitting radionuclides (Strontium-90) levels in plant-derived foods marketed in Italy. In:
Schneider K (ed) Advances in Vegetable Consumption and Health Research. Nova Science Publishers Inc, New York, pp 55-84

International Atomic Energy Agency - IAEA (1989) Measurement of radionuclides in food and the environment. https://inis.iaea.org/ collection/NCLCollectionStore/_Public/20/041/20041399.pdf. Accessed 19 June 2021

International Organization for Standardization (2010) ISO 21748:2010 - Guidance for the use of repeatability, reproducibility and trueness estimates in measurement uncertainty estimation.

International Organization for Standardization (2019) ISO 11929:2019 - Determination of the characteristic limits (decision threshold, detection limit and limits of the coverage interval) for measurements of ionizing radiation - Fundamentals and application - Part 1: Elementary applications.

Jeter HW, Grob B (1994) Determination of radiostrontium in milk using an extraction chromatography column. Radioactivity and Radiochemistry 5(3):8-17. https://www.osti.gov/biblio/160356. Accessed 19 June 2021

Jiang J, Davies A, Thorne K, Gilligan C (2017) Rapid analysis of ${ }^{89} \mathrm{Sr}$ and ${ }^{90} \mathrm{Sr}$ in nuclear forensics samples. J Radioanal Nucl $\mathrm{Ch}$ 311(2):1417-1425. https://doi.org/10.1007/s10967-016-5095-8

Kabai E, Hornung L, Savkin BT, Poppitz-Spuhler A, Hiersche L (2011) Fast method and ultra fast screening for determination of ${ }^{90} \mathrm{Sr}$ in milk and dairy products. Sci Total Environ 410:235-340. https:// doi.org/10.1016/j.scitotenv.2011.09.052

Kong X, Dang L, Shao X, Yin L, Ji Y (2018) Rapid method for determination of ${ }^{90} \mathrm{Sr}$ in biological samples by liquid scintillation counting after separation on synthesized column. J Environ Radioactiv 193-194:15-19. https://doi.org/10.1016/j.jenvrad. 2018.08.010

Landstetter C, Wallner G (2006) Determination of strontium-90 in deer bones by liquid scintillation spectrometry after separation on $\mathrm{Sr}-$ specific ion exchange columns. J Environ Radioact 87(3):315324. https://doi.org/10.1016/j.jenvrad.2005.12.008

Livens F (1990) Measurement of radionuclides in food and the environment - a guidebook. J Environ Radioactiv 11(2):201-202. https:// doi.org/10.1016/0265-931X(90)90062-Z

Lopes I, Madruga MJ, Mourato A, Abrantes J, Reis M (2010) Determination of ${ }^{90} \mathrm{Sr}$ in Portuguese foodstuffs. J Radioanal Nucl Ch 286(2):335-340. https://doi.org/10.1007/s10967-010-0714-2

Maxwell SL (2018) Rapid methods for actinide and Strontium-89,90 determination in urban matrices. Dissertation, University of South Carolina. https://doi.org/10.34917/13568585. https://digitalsch olarship.unlv.edu/cgi/viewcontent.cgi?article $=4291 \&$ context $=$ thesesdissertations. Accessed 19 June 2021

Maxwell SL, Culligan BK, Hutchison JB (2015) Rapid determination of ${ }^{90} \mathrm{Sr}$ in seawater samples. J Radioanal Nucl Ch 303(1):709-717. https://doi.org/10.1007/s10967-014-3391-8

Maxwell SL, Culligan B, Hutchison JB, Utsey RC, Sadowe R, McAlister DR (2016) Rapid method to determine actinides and ${ }^{89 / 90} \mathrm{Sr}$ in limestone and marble samples. J Radioanal Nucl Chem 310:377388. https://doi.org/10.1007/s10967-016-4783-8

Maxwell SL, Culligan BK, Noyes GW (2010) Rapid separation of actinides and radiostrontium in vegetation samples. J Radioanal Nucl Chem 286:273-282. https://doi.org/10.1007/s10967-010-0653-y

Maxwell SL, Culligan BK, Shaw PJ (2013) Rapid determination of radiostrontium in large soil samples. J Radioanal Nucl Ch 295(2):965-971. https://doi.org/10.1007/s10967-012-1863-2

Pan J, Emanuele K, Maher E, Lin Z, Healey S, Regan P (2017) Analysis of radioactive strontium- 90 in food by Čerenkov liquid scintillation counting. Appl Radiat Isotopes 126:214-218. https://doi. org/10.1016/j.apradiso.2017.01.034

Russell B, García-Miranda M, Ivanov P (2017) Development of an optimised method for analysis of $90 \mathrm{Sr}$ in decommissioning wastes by triple quadrupole inductively coupled plasma mass 
spectrometry. Appl Radiat Isotopes 126:35-39. https://doi.org/ 10.1016/j.apradiso.2017.01.025

Shuzhong Z, Shaoxian L, Zhenhong X (2015) Rapid determination of ${ }^{90} \mathrm{Sr}$ By TBP extraction. China Institute of Atomic Energy. https://inis.iaea.org/collection/NCLCollectionStore/_Public/32/ 065/32065262.pdf

Suomela J, Wallberg L, Melin J (1993) Methods for determination of strontium- 90 in food and environmental samples by Cerenkov counting. Swedish Radiation Protection Institute, Stockholm. https://www.stralsakerhetsmyndigheten.se/contentassets/27f6a 49844d6461195ae2fa2dd8e533a/199311-methods-for-deter mination-of-strontium--90-in-food-and-environmental-samplesby-cerenkov-counting. Accessed 19 June 2021

Surman JJ, Pates JM, Zhang H, Happel S (2014) Development and characterisation of a new $\mathrm{Sr}$ selective resin for the rapid determination of 90Sr in environmental water samples. Talanta 129:623628. https://doi.org/10.1016/j.talanta.2014.06.041

Temba ES, Júnior ASR, Amaral ÂM, Monteiro RP (2011) Separation and determination of ${ }^{90} \mathrm{Sr}$ in low-and intermediate-level radioactive wastes using extraction chromatography and LSC. J Radioanal Nucl Ch 290(3):631-635. https://doi.org/10.1007/ s10967-011-1327-0

Thomson M, Ellison SLR, Wood R (2002) Harmonized guidelines for single-laboratory validation of methods of analysis. Pure Appl Chem 74(5):835-855. https://doi.org/10.1351/pac200274050835

Triskem International (2016) Product sheet - SRResin. https://www. triskem-international.com/scripts/files/5f463447ad4026.94629 022/PS_SR-Resin_EN_160927.pdf. Accessed 19 June 2021
United States Environmental Protection Agency (2020) Radionuclide Basics: Strontium-90. https://www.epa.gov/radiation/radionucli de-basics-strontium-90\#strontiumhealth. Accessed 19 June 2021

Vaida N, Kim C-K (2010) Determination of radiostrontium isotopes: A review of analytical methodology. Appl Radiat Isotopes 68:23062326. https://doi.org/10.1016/j.apradiso.2010.05.013

World Health Organization and Food and Agriculture Organization - WHO/FAO (2011) Nuclear accidents and radioactive contamination of foods. https://www.who.int/docs/default-source/docum ents/radiation/nuclear-accidents-and-radioactive-contaminationof-foods.pdf?sfvrsn=8cff82e_1\&download=true. Accessed 19 June 201

World Health Organization - WHO (2005) Chernobyl: the true scale of the accident. https://www.who.int/news/item/05-09-2005-chern obyl-the-true-scale-of-the-accident. Accessed 19 June 2021

Wilken RD, Joshi SR (1991) Rapid methods for determining ${ }^{90} \mathrm{Sr},{ }^{89} \mathrm{Sr}$ and ${ }^{90} \mathrm{Y}$ in environmental samples: a survey. Radioact Radiochem 2(3):14-27

Publisher's Note Springer Nature remains neutral with regard to jurisdictional claims in published maps and institutional affiliations. 